\title{
Pathological Staging of Muscle Invasive Bladder Cancer. Is Substaging of pT2 Tumors Really Necessary?
}

\author{
Husnu Tokgoz, Kadir Turkolmez, Berkan Resorlu, Kenan Kose, Ozden Tulunay, Yasar Beduk
}

Departments of Urology, Biostatistics and Pathology, Ankara University School of Medicine, Ankara, Turkey

\begin{abstract}
Objective: Compare clinical outcomes in patients having urothelial tumors invading less than one half of the depth of bladder muscle and greater than one half of bladder muscle and, to determine various clinical variables as predictive factors for survival.

Materials and Methods: According to our inclusion criteria, 57 patients among cases with T2 bladder tumor were selected. Thirty-five patients (61.4\%) had pT2a (Group-1) and 22 patients (38.6\%) had pT2b (Group-2) muscle invasive tumors. Mean follow up time was 7.3 years for Group-1, and 6.1 years for Group-2. Multivariate analysis was performed in order to identify possible correlation of clinical variables like age, gender, grade of primary tumor, appearance of local and/ or distant metastasis with patient outcome.

Results: Five year recurrence-free and overall survival rates were $69.1 \%$ and $44.3 \%$ for patients with pT2a tumor, whereas these ratios were $66.1 \%$ and $43 \%$, respectively for patients with pT2b tumor $(p=0.896 ; p=0.975)$. Mean overall and progression-free survival times were $87.7 \pm 13.8$ and $116 \pm 13.12$ months for Group-1, while they were $73.8 \pm 13.7$ and $88.85 \pm$ 12.55 months for Group-2, respectively. On both univariate and multivariate analysis, age was noticed as an independent predictive factor for survival.

Conclusions: The depth of muscle invasion in bladder tumors has no prognostic significance. Recurrence of the disease either locally or at distant sites dramatically shortens patients' life. Being older than 60 years old during the time of radical surgery, is also a bad prognostic factor for overall and progression-free survival.
\end{abstract}

Key words: bladder neoplasms; TNM staging; neoplasm staging; survival

Int Braz J Urol. 2007; 33: 777-84

\section{INTRODUCTION}

Bladder carcinoma is the second most frequent urogenital tumor disease, more than $90 \%$ of which are transitional cell carcinoma (TCC). Nearly $30 \%$ of these tumors already are or will progress into muscle invasive tumor during their follow up (1).

After classification of bladder tumors in 1992, the AJCC (American Joint Committee on Cancer) and
UICC (Union Internationale Contre le Cancer) revised this TNM (tumor, lymph node and metastasis) bladder cancer staging system (2). They divided T2 tumors (muscle invasive tumor) into superficial (T2a, tumor invasion in less than one half of the depth of the bladder muscle) and deep (T2b, tumor invasion in more than one half of the depth of the bladder muscle). This need for sub grouping of T2 tumors arose after Jewett 
had reported a significant prognostic difference in bladder tumor patients with superficial and deep muscle invasion. Including 18 patients with muscle invasive bladder tumor, overall survival rate was $80 \%$ for T2a tumors, whereas it was only $8 \%$ for $\mathrm{T} 2 \mathrm{~b}$ tumors (3). Presently, since the 2002 version did not include any changes for the staging of bladder cancer, the use of the 1997 version is still encouraged in most guidelines.

After this study, several studies were conducted to compare clinical outcomes in terms of overall and recurrence-free survival rates (4-9). In contrast with the findings of Jewett in 1952, most of these studies were unable to find a prognostic difference between these subgroups. Last study published on this subject was by Yu et al. (10). They found significant difference when 10 year recurrence free survival rate in pT2a and pT2b cases were compared (79\% vs. 64\%). However, when they recompared after sub grouping as lymph node negative and positive, the difference disappeared.

The paper by Cheng et al., revealed somewhat more interesting findings. They evaluated the depth of invasion by micrometer measurement and its relation to clinical outcome (11). They found that tumor size, rather than the degree of depth of muscle invasion, had much more prognostic value in terms of cancer specific and distant metastasis free survival rates.

Similarly, the aim of our study was to evaluate the practicality of subdividing the muscle invasion into pT2a and PT2b in routine pathological examination and to assess if substaging of $\mathrm{T} 2$ bladder tumors is predictive for overall and recurrence free survival.

\section{MATERIALS AND METHODS}

We performed retrospective evaluation of 225 patients who had undergone radical cystectomy and bilateral pelvic lymphadenectomy for bladder urothelial carcinoma in our department between January 1990 and December 2005. The 1997 TNM system was used for staging. From records, we detected that all specimens had been assessed by the same pathology team. Multiple sections were obtained from the tumor, bladder wall and adjacent mucosa. In addition, sections from distant mucosa, regional lymph nodes and ureters were evaluated. In men, tissue blocks from the seminal vesicles and prostate were examined. In women, tissue blocks obtained from ovaries, uterus and vagina were examined. Palliative cystectomy cases; cases with upper urinary tract tumors and non-transitional cell carcinomas, were excluded. Also, cases with histological features of squamous differentiation were excluded.

We included cases with T2 urothelial carcinoma of the bladder which did not have lymph node metastasis on pathological evaluation of cystectomy specimens, and which were not given preoperative or early postoperative radio and/ or chemotherapy.

Finally, 57 patients were included in the study. Of these, 35 patients $(61.4 \%)$ had pT2a and 22 patients $(38.6 \%)$ had pT2b muscle invasive tumors. As mentioned previously, none of those patients had evidence of pelvic lymph node invasion.

Histological grading was performed according to the World Health Organization / International Society of Urologic Pathology Classification (12).

The patients were initially seen 2 months after surgery and then every 3 months for 2 years and every 6 months until disease progression or death. Each examination consisted of full physical examination, abdominal tomography, thorax X-rays and laboratory investigations like complete blood count, serum biochemical analysis.

The correlation of clinical and pathological variables with survival was investigated by the Cox proportional hazards test. Two, 3 and 5 year recurrence free and overall survival rates were compared between patients with pT2a (Group-1) and pT2b (Group-2) tumor. The Kaplan-Meier method was used to estimate progression free and cumulative survival probabilities. Differences between groups on the basis of progression free and overall survival rates were assessed with log-rank test. Multivariate analysis was performed using the Cox regression survival method. A two- tailed $p$ value of $<0.05$ was accepted as statistically significant. 


\section{RESULTS}

The mean patient age at surgery was 62.2 years for Group-1 and the mean follow up time was 7.3 years, while the mean age at surgery was 59.3 years in Group-2. Mean follow up time was 6.1 years for this group $(p=0.657 ; p=0.798)$. Demographic distributions of patients were given in Table-1. During follow-up, 19 patients died because of primary tumor progression. No patient had died from unrelated causes during follow up.

On both univariate and multivariate analysis, age was noticed as a predictive factor for survival. Patients younger than 60 years of age, had 2.5 times higher advantage for survival on univariate analysis $(\mathrm{p}=0.05$; hazard ratio $=2.45)$. On multivariate analysis, age ( $p=0.02$; Hazard ratio: 5.6), appearance of local and / or distant metastasis during follow up,

Table 1 - Distribution of cases by sex, age and grade.

\begin{tabular}{lcc}
\hline Characteristics & $\begin{array}{c}\text { Number of } \\
\text { pT2a (\%) }\end{array}$ & $\begin{array}{l}\text { Number of } \\
\text { pT2b (\%) }\end{array}$ \\
\hline Sex & & \\
$\quad$ Male & $32(91.4 \%)$ & $21(95.5 \%)$ \\
$\quad$ Female & $3(8.6 \%)$ & $1(4.5 \%)$ \\
Age & & \\
$\quad$ 60 or younger & $18(51.4 \%)$ & $14(63.6 \%)$ \\
$\quad$ Older than 60 & $17(48.5 \%)$ & $8(36.3)$ \\
$\begin{array}{l}\text { Grade } \\
\quad \text { Low }\end{array}$ & $21(60 \%)$ & $10(45.4 \%)$ \\
$\quad$ High & $14(40 \%)$ & $12(54.5 \%)$ \\
\hline
\end{tabular}

were also statistically significant effective factors on survival (Table-2).

Initial surgical management consisted of radical cystectomy, bilateral pelvic lymph node dissection and urinary diversion including neobladder replacement in 16 patients, continent urinary reservoir in 12 patients, ileal conduit in 28 patients and ureterocutaneostomy in 1 patient. Two, 3 and 5 year recurrence free survival rates were $83.7 \% ; 69.1 \%$ and $69.1 \%$, whereas $2,3,5$ year overall survival rates were $90.1 \% ; 71 \%$ and $44.3 \%$ for patients with pT2a tumor. Two, 3 and 5 year recurrence free survival rates for patients with pT2b tumor, were $73.5 \% ; 66.1 \%$ and $66.1 \%$, respectively. Overall survival rates for this group were $88.9 \%$; $64.6 \%$ and $43 \%$. Although, 2,3 and 5 year recurrence free and overall survival rates were lower in pT2b patients, both recurrence free survival and overall survival rates were statistically insignificant between Group-1 and 2 ( $\mathrm{p}=0.896 ; \mathrm{p}=$ 0.975) (Figure-1 and 2). On multivariate analysis, pT substaging (pT2b versus pT2a) had a hazard ratio greater than 1 (2.2), but the $\mathrm{p}$ value was again insignificant $(\mathrm{p}=0.142)$ (Table-2).

Mean survival time was $87.7 \pm 13.8$ months for Group-1, and 73.8 \pm 13.7 months for Group-2 ( $\mathrm{p}=$ $0.97)$. Mean progression-free survival time was 116 \pm 13.12 months for Group- 1 , while it was $88.85 \pm 12.55$ months for Group-2 ( $\mathrm{p}=0.53)$.

When we divided each group into two as patients with low grade and high grade tumors; mean survival times for patients with low and high grade tumors in Group-1, were $105.18 \pm 16.54$ and $37.8 \pm$ 5.57 months, respectively ( $\mathrm{p}=0.10)$. In Group- 2 , these were $53.33 \pm 17.06$ months for patients with low grade

Table 2 - Association of various variables with survival for patients with T2 bladder cancer on multivariate analysis.

\begin{tabular}{lccc}
\hline Parameter & p Value & Hazard Ratio & 95\% Confidence Interval \\
\hline Age $(>60$ versus $\leq 60)$ & 0.002 & 5.6 & $1.85-16.98$ \\
$\begin{array}{l}\text { Local and/ or distant metastasis } \\
\text { (positive versus negative) }\end{array}$ & 0.000 & 31.4 & $6.75-146.16$ \\
pT stage (pT2b versus pT2a) & 0.142 & 2.2 & $0.75-6.85$ \\
\hline
\end{tabular}




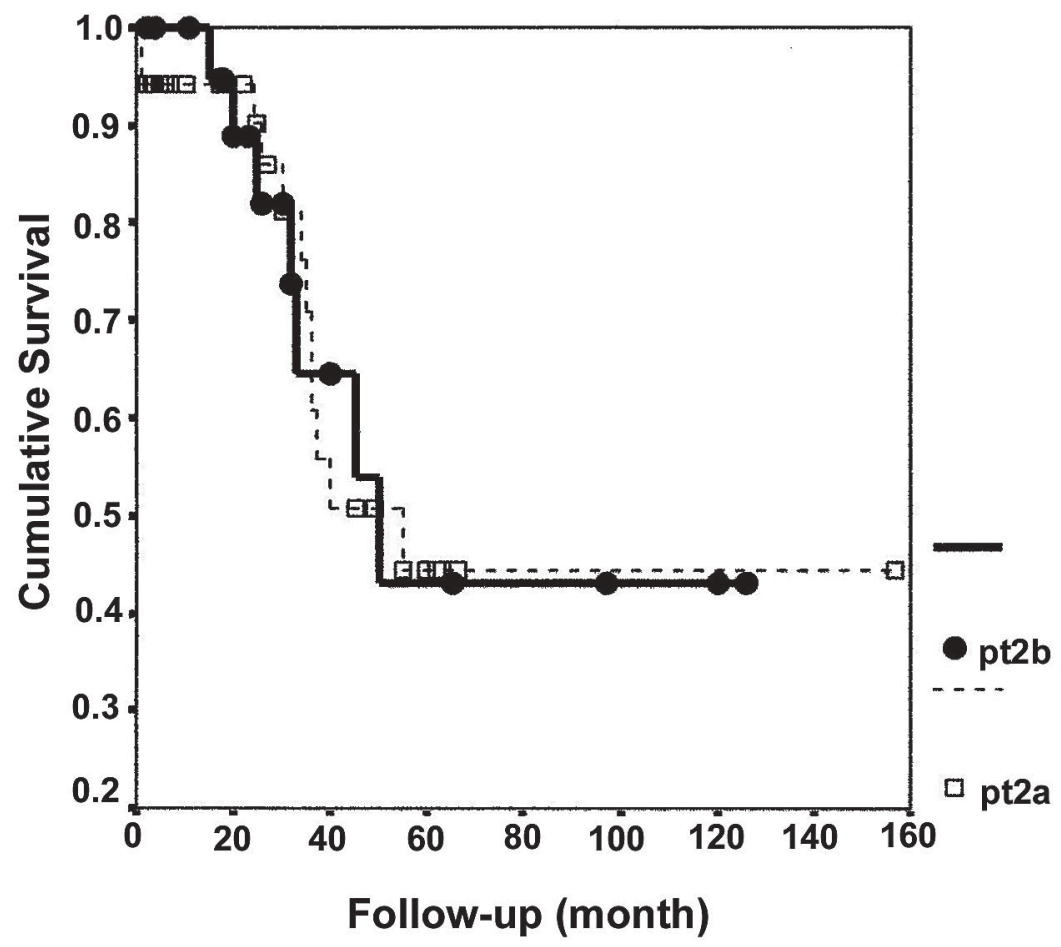

Figure 1 - Kaplan-Meier curve demonstrating cumulative survival in patients with pT2a and pT2b bladder cancer.

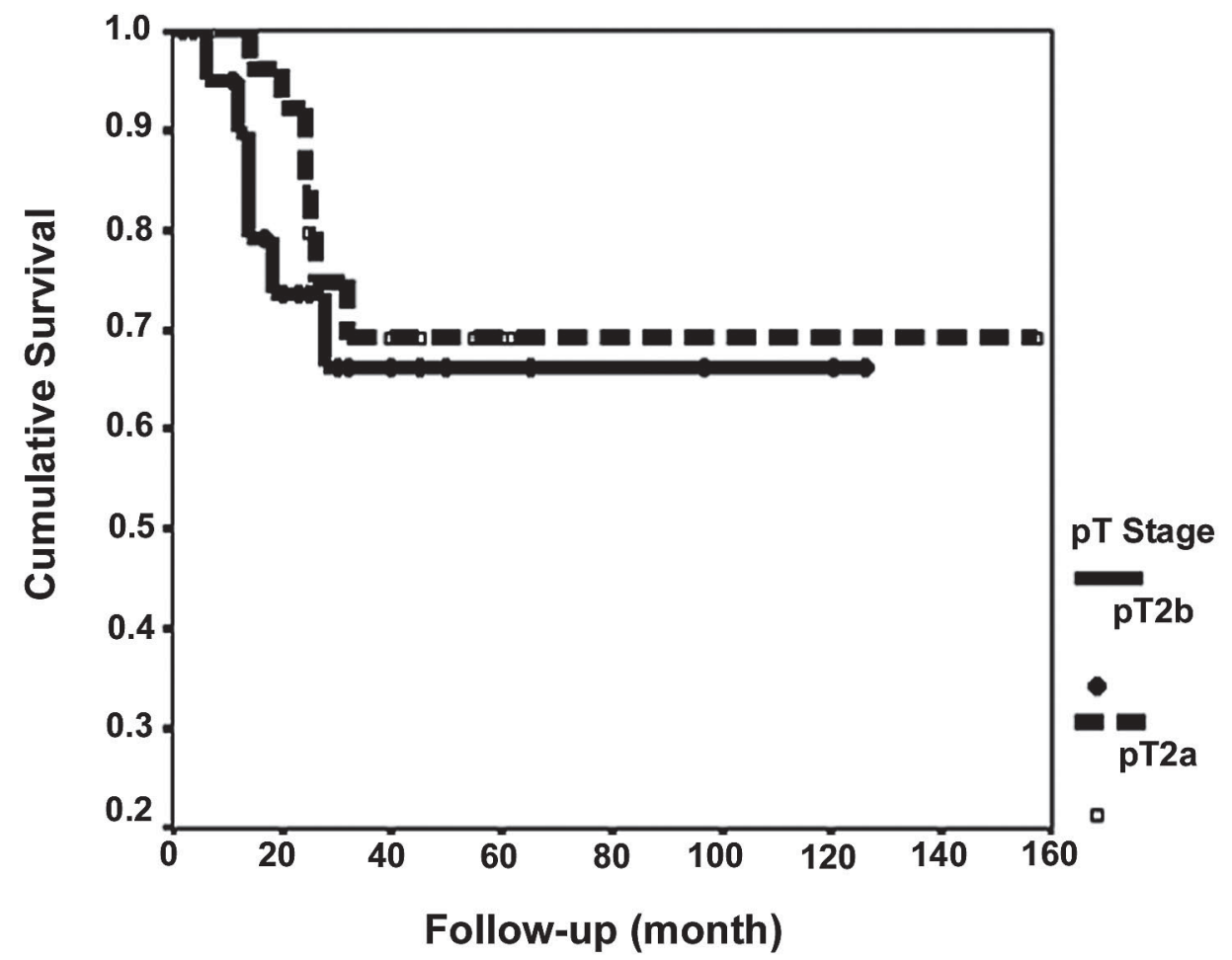

Figure 2 - Kaplan-Meier curve demonstrating progression-free survival in patients with pT2a and pT2b bladder cancer. 
tumors and $84.4 \pm 16.4$ months for patients with high grade tumors $(\mathrm{p}=0.32)$. Although the differences seem great especially in Group-1, no statistical significance was present.

Local pelvic recurrence or distant metastasis developed in 13 of 57 patients $(22.8 \%)$ with pT2 disease. Seven (20\%) out of 13 patients who showed progression of the primary tumor during follow up, were belong to Group-1 (distant metastasis in 6; both local recurrence and distant metastasis in 1 case), whereas 6 patients $(27.2 \%)$ in Group-2 showed progression of primary tumor (local pelvic recurrence in 1; distant metastasis in 5 cases). There was no statistically significant difference when groups were compared $(\mathrm{p}=0.496)$.

None of the variables, including gender and primary tumor grade were significantly associated with patient outcome. Thus, none of them were believed to be predictive of patient survival.

\section{COMMENTS}

Presently, TNM staging system revised in 1997 seems to provide the most valuable prognostic information in patients with transitional cell carcinoma of the bladder. Superficial (T1), muscle invasive (T2) and perivesical invasive (> T2) urothelial tumors have significantly different 5 year recurrence free and survival rates. Nicely, T1 tumors form the major and best prognostic group. Only $25 \%$ of these cases do not survive 5 years (13). For T2 tumors, 5 year survival rates were given as approximately 60\% (40-70\%) (49). Nevertheless, Skinner and Herr, reported that urothelial cancers invading perivesical fat tissue had much worse prognosis than those not invading the perivesical fat tissue (14-17).

For high grade, non metastatic, muscle invasive urothelial tumors, radical cystectomy is accepted as the gold standard treatment. Although it is a major surgical procedure including reconstructive techniques for neobladder replacement, this operation provides high 5 year survival rates. In addition, cystectomy with pelvic lymph node dissection allows the clinician to know accurate pathological staging of the primary tumor, and aids in further treatment planning, including adjuvant radio and / or chemotherapy.

For organ confined lymph node negative T2 tumors, May et al., reported a 74\% progression free 5 year survival rate, which was very close to the ratios given for T1 tumors (18). In a study by Wishnow and colleagues, 5 year mean survival and disease free survival rates after radical cystectomy, were 82 and $77 \%$, respectively for patients with Stage B disease (19). In 2001, Stein et al. presented their long term surgical experience and patient outcomes in 1054 cases treated with radical cystectomy for invasive transitional cell carcinoma of the bladder (1). Their 5 and 10 year recurrence free survival rates for organconfined lymph node negative tumors were, $85 \%$ and $82 \%$, respectively. However, it was not the case for lymph node positive cases. Five year recurrence free and overall survival rates were, $35 \%$ and $31 \%$, respectively for those patients.

In last years, major criticism on TNM system, was focused on if substaging of T2 tumors was really necessary. The findings of the studies evaluating the correlation of depth of muscle invasion with patient survival, indicate that this parameter is of no prognostic value (4-9). For this reason, some of the urooncologists offer the removal of substaging of $\mathrm{T} 2$ bladder cancers from TNM systems.

Although our study was retrospective, parameters like age, gender, and mean follow up times were similar in each group. Tumor progression rates for Group-1 and 2 were $20 \%$ and $27.2 \%$ respectively, which were also close $(p>0.05)$. Grade of the primary tumor was not correlated with survival of patients in both groups. Statistically significant difference was only observed when mean survival times of patients who developed progression of the disease were compared with survival times of the cases who were recurrence or metastasis free during their follow up.

Instead of substaging, Cheng et al., mentioned the prognostic value of primary tumor size (11). Actually, we had problems in accurate evaluation of primary tumor size, since they were not always mentioned in pathology reports. So, we were not able to include this parameter for survival analysis. Cheng and associates found lower results than our study, revealing $62 \% 5$ year survival rate for patients with 
pT2a tumors and $56 \%$ for patients with pT2b tumors (11). But, they included node positive cases. After they divided study population according to their lymph node status, they reported 10 year recurrence free survival as $74 \%$ for lymph node negative cases.

We were interested in age as an independent predictive factor for survival, and we observed statistically significant difference in the 6th decade. This means that, for bladder tumor patient, being older than 60 years old at the time of radical surgery is a bad prognostic factor for both progression-free and overall survival.

One should reasonably think that the appearance of local and / or distant metastasis during follow-up, is a progression of the disease, not a real predictive factor. So, high correlation of this variable with survival might be expected. But, it is not the case for age. As Cheng et al. insisted on the importance of primary tumor size, we aimed to take the attention of uro-oncologists on the predictive value of age (11). Most prominent difference was observed in the 6th decade in our study. Thus, we have taken the age 60 as a cut-off point.

Regarding that all cases in our study population were lymph node negative, the rates $\mathrm{Yu}$ and colleagues reported, were higher than our results $(69.1 \%$ vs. $66.1 \%)$. They reported 10 year recurrence free survival rate for node negative pT2a disease as $84 \%$ and for pT2b disease as $74 \%$ (10).

But, we cannot ignore the effect of substaging on survival. In our study, although the $\mathrm{p}$ value was insignificant; odds ratio of higher than 1 (2.2) on multivariate analysis, suggest possible effect of pT substaging on cancer specific survival of these patients. Similarly, in the study by Cheng, 10 year survival rates were lower in cases with pT2b disease compared with pT2a disease (51\% vs. $48 \%$ ). But, the p value was again insignificant (11). When they re-compared 10 year cancer specific survival rates in lymph node negative cases between the 2 groups, they found a risk ratio of 2 (pT2b versus pT2a); a value which was so close to ours (2.2).

Limitations of the study should be discussed. First of all, our sample size is relatively small. But, in order to form homogenous study groups, we excluded cases; with histological features of squamous differentiation, having nodal involvements on pathological evaluation of cystectomy specimens, and who were given preoperative or early postoperative radio and / or chemotherapy. We hope in the near future, prospective studies with larger series, will be published and, will give more valuable data.

\section{CONCLUSIONS}

We think that the depth of muscle invasion in bladder tumors has no prognostic significance. But, we cannot fully exclude its role on cancer specific survival. In addition, the results underline the importance of patient age at the time of surgery, as age was found an independent prognostic factor for both overall and progression-free survival.

\section{CONFLICT OF INTEREST}

None declared.

\section{REFERENCES}

1. Stein JP, Lieskovsky G, Cote R, Groshen S, Feng AC, Boyd S, et al.: Radical cystectomy in the treatment of invasive bladder cancer: long-term results in 1,054 patients. J Clin Oncol. 2001; 19: 666-75.

2. Fleming ID, Cooper JS, Henson DE: American Joint Committee on Cancer, Cancer Staging Manual. (5th ed,). Philadelphia, Lippincott-Raven. 1997; pp. 241-3.

3. Jewett HJ: Carcinoma of the bladder: influence of depth of infiltration on the 5-year results following complete extirpation of the primary growth. J Urol. 1952; 67: 672-80.

4. Ghoneim MA, el-Mekresh MM, el-Baz MA, el-Attar IA, Ashamallah A: Radical cystectomy for carcinoma of the bladder: critical evaluation of the results in 1,026 cases. J Urol. 1997; 158: 393-9.

5. Pollack A, Zagars GK, Cole CJ, Dinney CP, Swanson DA, Grossman HB: The relationship of local control to distant metastasis in muscle invasive bladder cancer. J Urol. 1995; 154: 2059-63; discussion 2063-4.

6. Roehrborn CG, Sagalowsky AI, Peters PC: Long-term patient survival after cystectomy for regional metastatic transitional cell carcinoma of the bladder. J Urol. 1991; 146:36-9. 
7. Sorensen BL, Soeborg Ohlsen A, Barlebo H: Carcinoma of the urinary bladder. Clinical staging and histologic grading in relation to survival. Scand J Urol Nephrol. 1969; 3: 189-92.

8. Cuesta JA, Chapado MS, Cid MG, Corral NF, Pontes EJ, Grignon DJ: Survival of patients with stage T2-3a bladder cancer treated by radical cystectomy. Arch Esp Urol. 1997; 50: 17-25.

9. Pagano F, Bassi P, Galetti TP, Meneghini A, Milani C, Artibani W, et al.: Results of contemporary radical cystectomy for invasive bladder cancer: a clinicopathological study with an emphasis on the inadequacy of the tumor, nodes and metastases classification. J Urol. 1991; 145: 45-50.

10. Yu RJ, Stein JP, Cai J, Miranda G, Groshen S, Skinner DG: Superficial (pT2a) and deep (pT2b) muscle invasion in pathological staging of bladder cancer following radical cystectomy. J Urol. 2006; 176: 493-8; discussion 498-9.

11. Cheng L, Neumann RM, Scherer BG, Weaver AL, Leibovich BC, Nehra A, et al.: Tumor size predicts the survival of patients with pathologic stage T2 bladder carcinoma: a critical evaluation of the depth of muscle invasion. Cancer. 1999; 85: 2638-47.

12. Epstein JI, Amin MB, Reuter VR, Mostofi FK: The World Health Organization/International Society of
Urological Pathology consensus classification of urothelial (transitional cell) neoplasms of the urinary bladder. Bladder Consensus Conference Committee. Am J Surg Pathol. 1998; 22: 1435-48.

13. Angulo JC, Lopez JI, Grignon DJ, Sanchez-Chapado M: Muscularis mucosa differentiates two populations with different prognosis in stage T1 bladder cancer. Urology. 1995; 45: 47-53.

14. Herr HW: Staging invasive bladder tumors. J Surg Oncol. 1992; 51: 217-20.

15. Skinner DG: Current perspectives in the management of high-grade invasive bladder cancer. Cancer. 1980; 45: 1866-74.

16. Herr HW: A proposed simplified staging system of invasive bladder tumors. Urol Int. 1993; 50: 17-20.

17. Skinner DG: Current state of classification and staging of bladder cancer. Cancer Res. 1977; 37: 2838-42.

18. May M, Helke C, Nitzke T, Vogler H, Hoschke B: Survival rates after radical cystectomy according to tumor stage of bladder carcinoma at first presentation. Urol Int. 2004; 72: 103-11.

19. Wishnow KI, Ayala AJ, Levinson AK, Logothetis CJ, Johnson DE, Swanson DA, et al.: Stage B (P2/ 3A/ N0) transitional cell carcinoma of bladder highly curable by radical cystectomy. Urology 1992; 39: 126.

$\overline{\text { Accepted after revision: }}$

August 8, 2007

\author{
Correspondence address: \\ Dr. Hüsnü Tokgöz \\ Çukuranbar Mah. 41. Cad. \\ No: 2 / 3506520 \\ Balgat, Ankara, Turkey \\ Tel: +90312-2847285 \\ E-mail:h_tokgoz@hotmail.com
}

\section{EDITORIAL COMMENT}

In 1952, Jewitt proposed the subclassification of T2 (prior stage B) tumors based on their depth of involvement of the muscular wall - T2a (B1) involving the inner half and T2b (B2) infiltrating the outer half based on survival differences observed in just 18 patients. Over the ensuing decades, increasing 
worldwide experience suggested that perhaps such substaging may not be in fact predictive leading Jewitt to revise his comments stating in 1978 that "the arbitrary dividing line drawn 30 years ago at the halfway level to separate B1 and B2 was too superficial." His remarks seem to also acknowledge the greater relevance of deeper invasion - i.e. involvement of the perivesical soft tissue - as having worse diseaseassociated outcomes.

The present study supports this contention by failing to demonstrate any survival differences in bladder cancer patients when stratified by depth of muscle-invasion - T2a versus T2b. These results support the recent findings Cheng et al. from the Mayo Clinic. Furthermore, although Yu et al. recently demonstrated decreased survival in T2b vs. T2a patients, these differences disappeared when patients were re-analyzed according to lymph node status.

The ongoing study to help identify which clinical and pathological parameters have a relevant impact on a patient's prognosis remains an important and worthy research endeavor. However, the answers may ultimately lie beyond the clinical and histological findings alone, and are likely to be found in the molecular and genetic signatures of tumors occurring at the cellular level. Tumor suppressor genes (e.g. p53 and $\mathrm{Rb}$ ), proliferative indices (Ki-67), urinary growth factors (e.g. epidermal growth factor, basic fibroblast growth factor, and CD44), matrix metalloproteinses (e.g. MMP-9), and urinary plasminogen activators are just some of the indicators that one day may prove to be valuable adjuncts in the management of urothelial cancers.

These include blood group antigens (e.g., LewisX), tumor suppressor genes (e.g. p53 and Rb), proliferative indices (Ki-67), urinary growth factors (e.g. epidermal growth factor, basic fibroblast growth factor, and CD44), matrix metalloproteinses (e.g. MMP-9), and urinary plasminogen activators. One day such indicators may prove to be valuable adjuncts to stage and grade in the management of noninvasive urothelial cancers.

Dr. Raj S. Pruthi

Associate Professor of Surgery/Urology

Director of Urologic Oncology

The University of North Carolina at Chapel Hill

Chapel Hill, North Carolina, USA

E-mail:rpruthi@med.unc.edu 